Proceedings of the LIV Zakopane School of Physics, Breaking Frontiers, Zakopane, Poland, May 21-25, 2019

\title{
Magnetic Properties of Interference Pulsed Laser Heated Fe-Based Amorphous Ribbons
}

\author{
O. Czyż ${ }^{a, *}$, J. Kusiński ${ }^{a}$, A. Radziszewska ${ }^{a}$, M. KąC ${ }^{b}$ And R. Ostrowski ${ }^{c}$ \\ ${ }^{a}$ AGH University of Science and Technology, Kraków, Poland \\ ${ }^{b}$ Institute of Nuclear Physics Polish Academy of Sciences, PL-31342 Kraków, Poland \\ ${ }^{c}$ Military University of Technology, Warszawa, Poland
}

\begin{abstract}
The influence of pulsed laser interference heating on structure and magnetic properties of Fe-based (FeSiB) amorphous ribbons is reported. This form of heating results in periodically placed laser heated micro-areas. In these, changes in microstructure and magnetic properties occur and the results are compared with those for samples after conventional annealing at $600^{\circ} \mathrm{C}$. Electron microscopy showed that the laser beam energy causes partial crystallization of the amorphous material, which affects magnetic properties. Magnetic hysteresis loop measured by SQUID magnetometer showed that saturation magnetization $\sigma$ lowered after both treatment (i.e., conventional annealing and laser heating) in comparison to amorphous ribbons (where the range of $\sigma$ was from 155 to $185 \mathrm{emu} / \mathrm{g}$ ), except for $\mathrm{FeCuSiB}$ alloy after laser heating. Magnetic structure of all the samples was also observed by magnetic force microscopy. While no signal was detected in the as-received amorphous ribbons, conventional annealing and laser heating resulted in strong magnetic signals.
\end{abstract}

DOI: 10.12693/APhysPolA.137.70

PACS/topics: FeSiB, magnetic properties, SQUID, Magnetic force microscopy, laser heating

\section{Introduction}

Fe-based (FeSiB) amorphous ribbons are characterized by an unusual combination of magnetic, electric, mechanical, and chemical properties. They possess high yield strength, corrosion and wear resistance. Due to their soft magnetic properties [1-5], including high saturation magnetization and near-zero coercivity, these materials are used in fabrication of magnetic cores, wires and shields, as well as detectors and band-pass filters [2]. The best magnetic properties are for alloys that are not strictly amorphous, but with nanometric grain size, since increased of their sizes further increases coercivity. Therefore, it is necessary to produce nanocrystalline alloys [5] and accordingly many unconventional techniques are investigated to obtain nanocrystalline structure out of amorphous alloys [6-11].

Pulsed laser interference patterning, known also as direct laser interference lithography (DLIL), can produce very precise periodic nanostructures, uniformly distributed over large areas. It is a fast-developing field of investigations with applications in many technological areas [12-14]. Our previous investigation (only for $\mathrm{FeSiB}$ ribbons) showed that this technique creates periodically placed laser heated dots, in which structure and magnetic properties changes occur $[10,11]$.

The present work is focused on structure and magnetic properties of Fe-based $(\mathrm{FeSiB}, \mathrm{FeCuSiB}$, and

\footnotetext{
*corresponding author; e-mail: olafczyz@agh.edu.pl
}

$\mathrm{FeCuNbSiB}$ ) amorphous alloys after interference pulsed laser heating. The results are compared to the relevant properties of amorphous ribbons after their conventional crystallization by annealing.

\section{Experimental procedure}

The investigated materials were in the form of $30 \mu \mathrm{m}$ thick and $25 \mathrm{~mm}$ or $10 \mathrm{~mm}$ wide ribbons. Their chemical compositions measured by Inductively Coupled Plasma - Optical Emission were: $\quad \mathrm{Fe}_{80} \mathrm{Si}_{11} \mathrm{~B}_{9}$, $\mathrm{Fe}_{77} \mathrm{Cu}_{1} \mathrm{Si}_{13} \mathrm{~B}_{9}$, and $\mathrm{Fe}_{75} \mathrm{Cu}_{1} \mathrm{Nb}_{2} \mathrm{Si}_{13} \mathrm{~B}_{9}$. Pulsed laser interference heating (PLIH) was carried out with Nd:YAG laser with basic wavelength $1064 \mathrm{~nm}$ and $120 \mathrm{~mJ}$ energy. Pulse time duration was $8 \mathrm{~ns}$ and frequency was $1 \mathrm{~Hz}$. Interference pattern resulted from the application of quartz tetrahedral prism. During PLIH a number of consecutive laser pulses in the same area varied from 50 to 500 . Conventionally annealed samples were prepared by heating to $600^{\circ} \mathrm{C}$ at $20^{\circ} \mathrm{C} / \mathrm{min}$ rate and slow cooling to room temperature. Structure of amorphous, laser heated, as well as annealed samples were investigated by scanning (SEM-FEI Inspect S50) and transmission electron (TEM-Jeol JEM 2010-ARP) microscopy.

Magnetic hysteresis loops in the samples of the form of discs (3 mm diameter) were measured using SQUID magnetometer (Quantum Design, MPMS). Magnetic moment was determined with the accuracy of $2 \%$. Magnetization at the sample surface was detected by magnetic force microscopy (MFM; Veeco Dimension ${ }^{\circledR}$ Icon $^{\text {TM }}$ SPM instrument). 


\section{Results and discussion}

\subsection{Microstructure}

Pulsed laser interference heating led to the creation of periodically placed laser heated microareas/dots, as shown in Fig. 1. The distance between laser dots was about $16 \mu \mathrm{m}$. FeSiB alloys (Fig. 1a, b) and $\mathrm{FeCuSiB}$ alloys (Fig. 1c, d) had a smoother and less damaged surface then FeCuNbSiB alloy (Fig. 1e, f). Magnification of the laser heated microarea showed ripples, as marked in Fig. 1b, d, f. The distance between the ripples was equal to the wavelength of Nd:YAG laser beam $~ 1064 \mathrm{~nm}$. According to Jia et al. [15], the ripples originate from the interference between the incident laser light and the scattered tangential wave. This tangential wave is a result of incident laser light partially scattered by surface defects. Transmission electron microscopy showed a fine-grained structure of the FeSiB alloy after PLIH, as shown in Fig. 2. Selected area diffraction pattern (inset in Fig. 2a) and dark field image (Fig. 2b) indicate the presence of $\alpha-\mathrm{Fe}(\mathrm{Si})$ nanocrystals that form aggregates of the size up to $400 \mathrm{~nm}$.

Conventional annealing of the $\mathrm{FeSiB}$ amorphous alloy led to the creation of a dendritic microstructure with variable dendrite size (Fig. 3a). Cu alloy addition results in forming individual $\alpha-\mathrm{Fe}(\mathrm{Si})$ nanocrystals embedded in the amorphous matrix (Fig. 3b, c), while $\mathrm{Nb}$ leads to a still decreased crystal size (Fig. 3c).
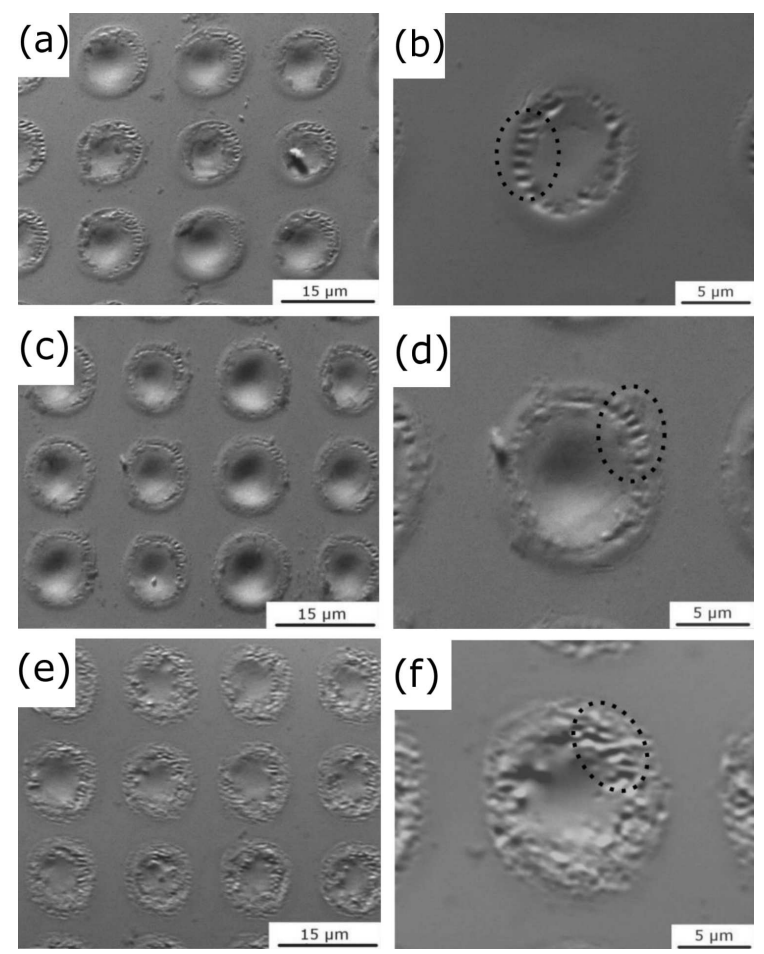

Fig. 1. SEM images of $\mathrm{FeSiB}(\mathrm{a}, \mathrm{b}), \mathrm{FeCuSiB}$ (c, d) and $\mathrm{FeCuNbSiB}(\mathrm{e}, \mathrm{f})$ alloys after PLIH with $120 \mathrm{~mJ}$ energy and 500 laser pulses. Ripples (see main text) are marked by dotted lines.

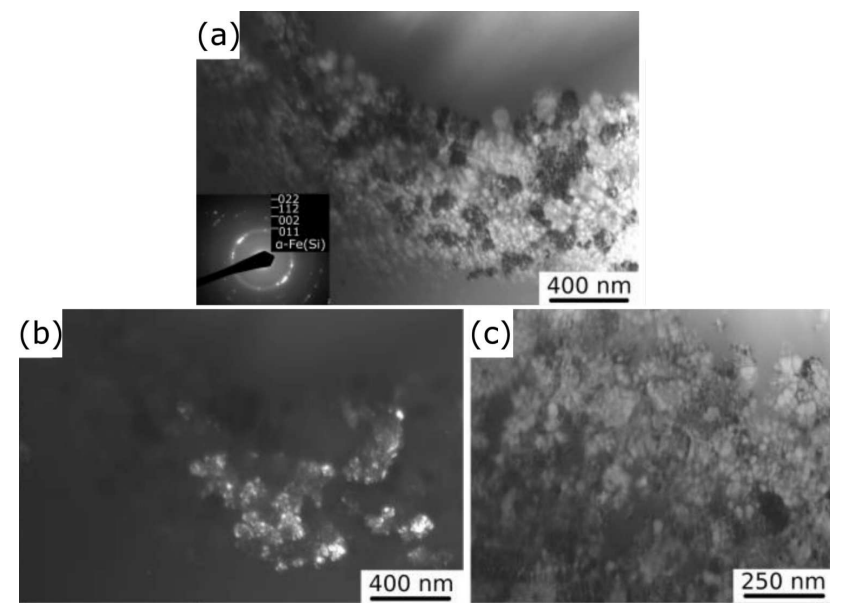

Fig. 2. TEM images of FeSiB alloy after PLIH with $120 \mathrm{~mJ}$ energy and 500 laser pulses: (a) bright field with diffraction, (b) darkfield, (c) magnification of (a) image.

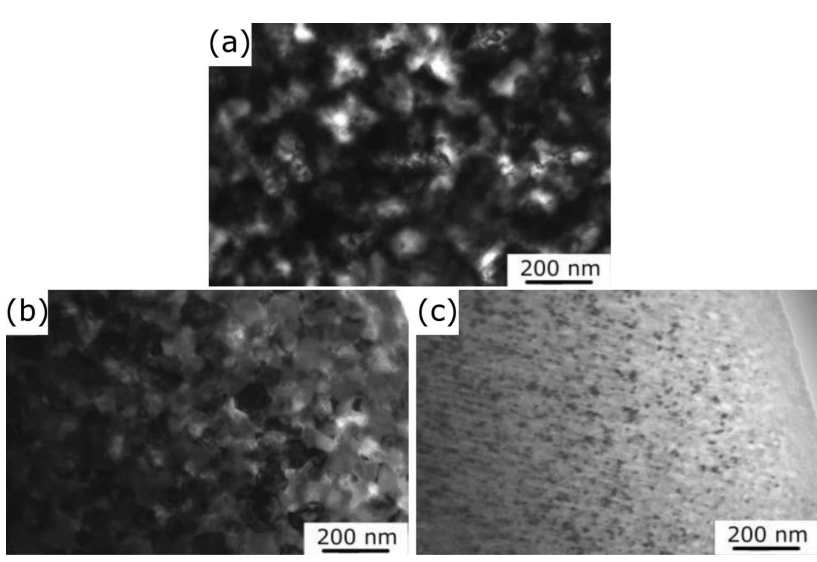

Fig. 3. TEM images of alloys after crystallization at $600^{\circ} \mathrm{C}$ : (a) FeSiB, (b) FeCuSiB, (c) FeCuNbSiB.

\subsection{Magnetic properties}

Magnetization process of amorphous ribbons showed a hard axis of magnetization perpendicular to the sample surface and the easy axis of magnetization parallel to the sample surface (in-plane anisotropy), as shown in Fig. 4. Ribbons saturate at about 300 Oe along the easy axis, while $30 \mathrm{kOe}$ is needed to saturate the samples along hard axes. Very soft magnetic properties of amorphous ribbons were revealed by magnetic hysteresis loop measurements, as shown in Fig. 5. FeSiB alloy has highest saturation magnetization $183 \mathrm{emu} / \mathrm{g}$, while the alloy additions of $\mathrm{Cu}$ and $\mathrm{Nb}$ decreased saturation magnetization to $163 \mathrm{emu} / \mathrm{g}$ and $155 \mathrm{emu} / \mathrm{g}$, respectively, (Fig. 5b). Coercivity for each alloy was the same, 20 Oe (Fig. 5c).

Crystallization by conventional annealing decreased saturation magnetization, as shown in Fig. 6, except for the $\mathrm{FeCuNbSiB}$ alloy. For the base alloy $\mathrm{FeSiB}$ it was $180 \mathrm{emu} / \mathrm{g}$, and $156 \mathrm{emu} / \mathrm{g}$ for $\mathrm{FeCuSiB}$ alloy 


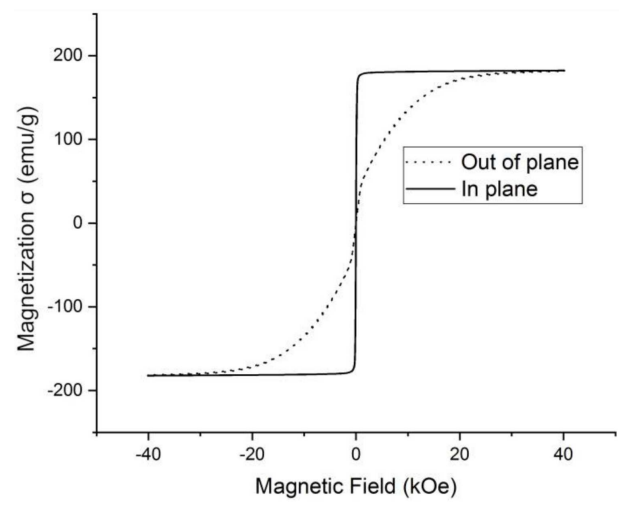

Fig. 4. Magnetic hysteresis loop of FeSiB amorphous alloy.
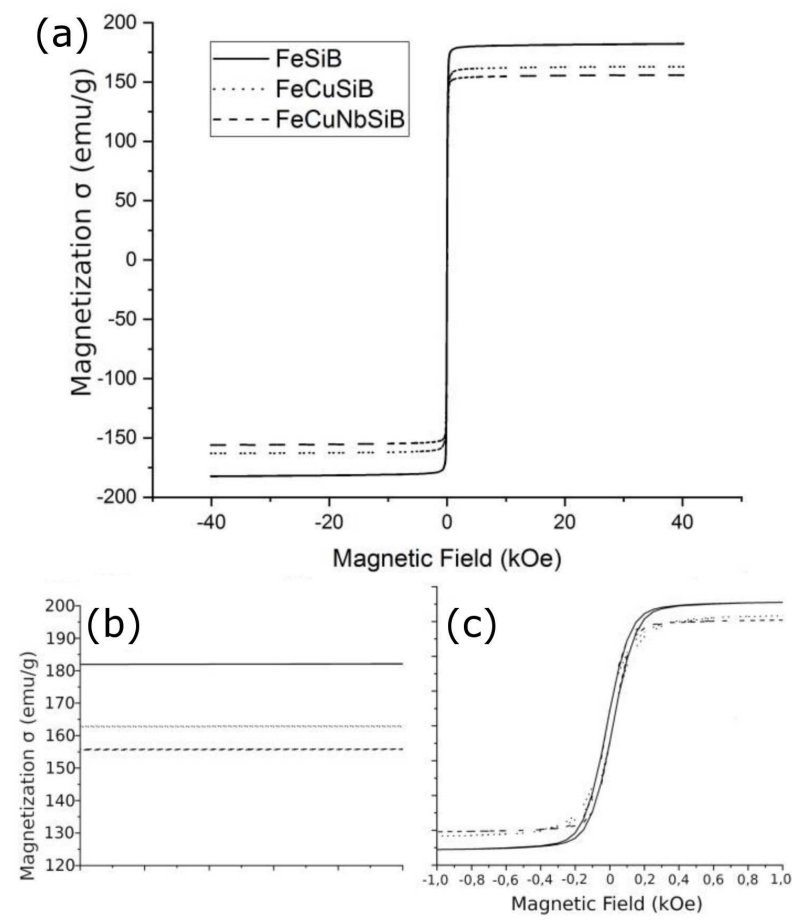

Fig. 5. SQUID measurements of Fe-based amorphous alloys: (a) magnetic hysteresis loops, (b) magnification of the saturation area, (c) magnification of the coercivity area.

(Fig. 6b). In these two cases, crystallization by conventional annealing led to an increase in coercivity to 40 Oe (Fig. 6c), while for the alloy with niobium it remained unchanged with values of 20 Oe. The reason for this distinct behaviour may be due to change in crystal sizes. As Herzer [5] showed, increasing grain size led to increased coercivity of Fe-based nanocrystalline alloys, as observed here for $\mathrm{FeSiB}$ and $\mathrm{FeCuSiB}$ alloys. Niobium addition strongly decreases the size of crystals as discussed above. After crystallization, the crystals in FeCuNbSiB alloy were smaller than $50 \mathrm{~nm}$ (Fig. 3b, 3c).
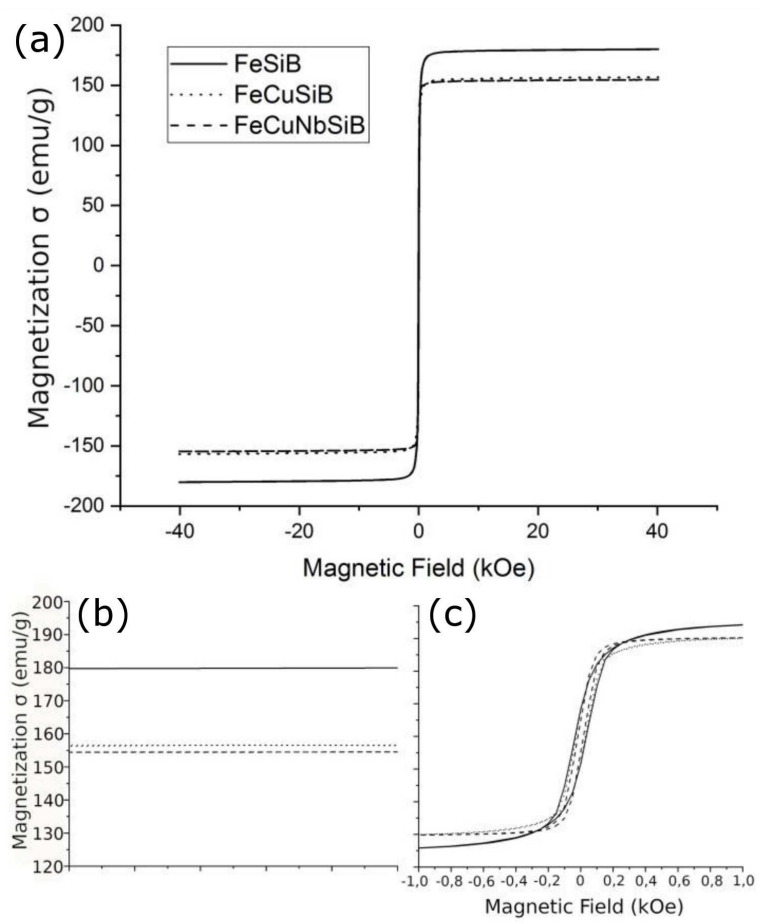

Fig. 6. SQUID measurements of Fe-based alloys after crystallization at $600^{\circ} \mathrm{C}$ : (a) magnetic hysteresis loops, (b) magnification of the saturation area, (c) magnification of the coercivity area.

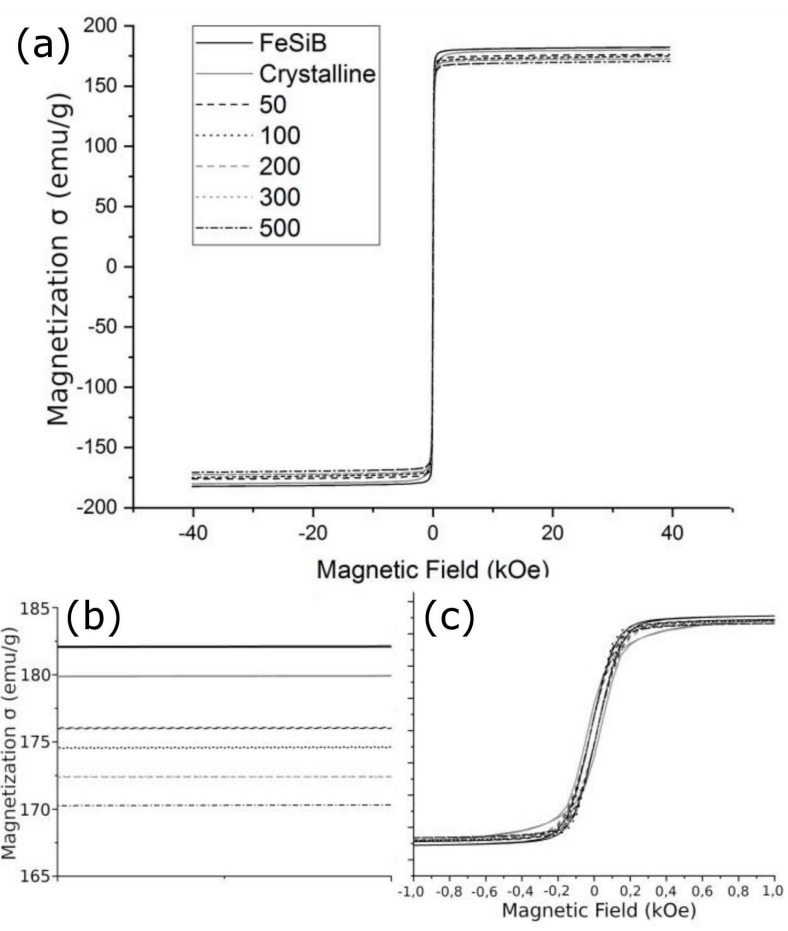

Fig. 7. SQUID measurements of FeSiB alloy after PLIH with $120 \mathrm{~mJ}$ energy and a different number of laser pulses: (a) magnetic hysteresis loops, (b) magnification of the saturation area, (c) magnification of the coercivity area. 


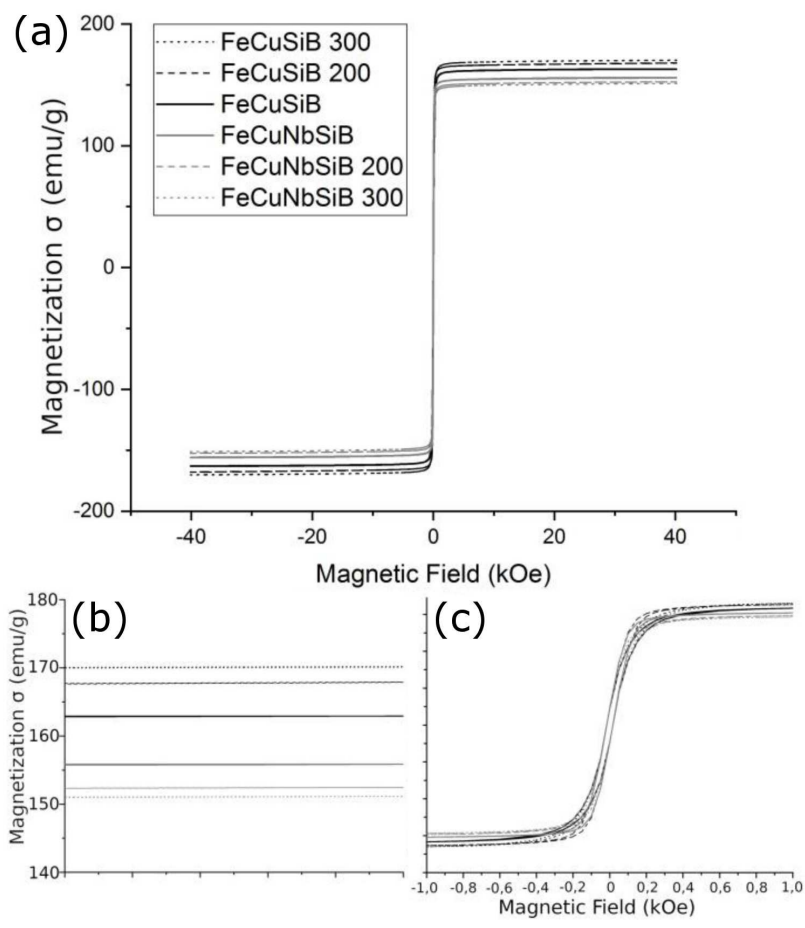

Fig. 8. SQUID measurements of Fe-based alloys after PLIH with $120 \mathrm{~mJ}$ energy and a different number of laser pulses: (a) magnetic hysteresis loops, (b) magnification of the saturation area, (c) magnification of the coercivity area.

Pulsed interference laser heating led to a decreased saturation magnetization of all the alloys without a change of coercivity, as shown in Fig. 7. In the case of FeSiB and $\mathrm{FeCuNbSiB}$ alloys, increasing the number of laser pulses led to still lower saturation magnetization (Figs. 7b and $8 \mathrm{~b}$, respectively), unlike in $\mathrm{FeCuSiB}$, where consecutive laser pulses increased saturation magnetization with no influence on coercivity (Fig. 7c). For all the alloys repeated pulsed laser interference heating (Fig. 8c) did not affect their coercivity.

\subsection{Magnetic Force Microscopy}

Magnetization at the sample surface was observed by magnetic force microscopy. Since the microscope chamber is equipped with a magnetic shield, a possible external magnetic field at the sample is almost zero and the detected magnetic signal came only from the sample.

The amorphous alloys gave almost zero magnetic signals, as shown in Fig. 9, and it was in accord with nearly negligible coercivity. Therefore, only noise from topography was observed on magnetic forces images.

PLIH changed the microstructure of these materials only in the laser heated dots. In particular, MFM measurements of FeSiB alloy detected magnetization only in microareas affected by the laser beam, as shown in Fig. 10, because higher coercivity was found in crystallized materials, as mentioned above. Magnetic forces and the ensuing signal are strengthened in the loops surrounding each laser affected micro-area. Magnetic signal got stronger with higher number of laser pulses. Since an increasing number of laser pulses also results in diminishing saturation magnetization (Fig. 5b), but not a change in coercivity (Fig. 5c), these two facts suggest that the volume of the laser affected material increases in the consecutive laser shots rather than the nanocrystal size.

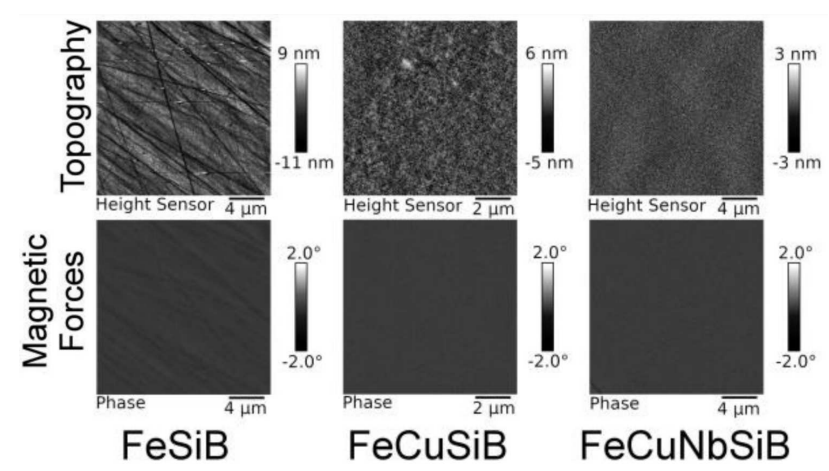

Fig. 9. Topography and magnetic forces images of Febased amorphous alloys.

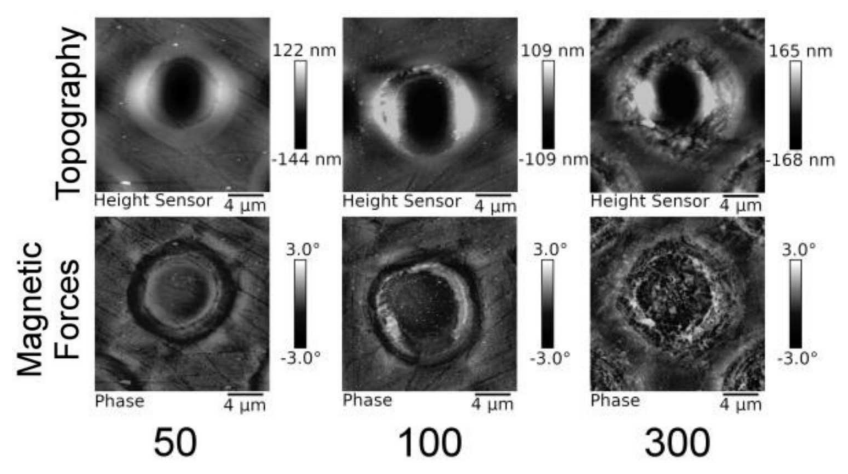

Fig. 10. Topography and magnetic forces images of Fe$\mathrm{SiB}$ alloy after PLIH with $120 \mathrm{~mJ}$ energy and a different number of laser pulses.

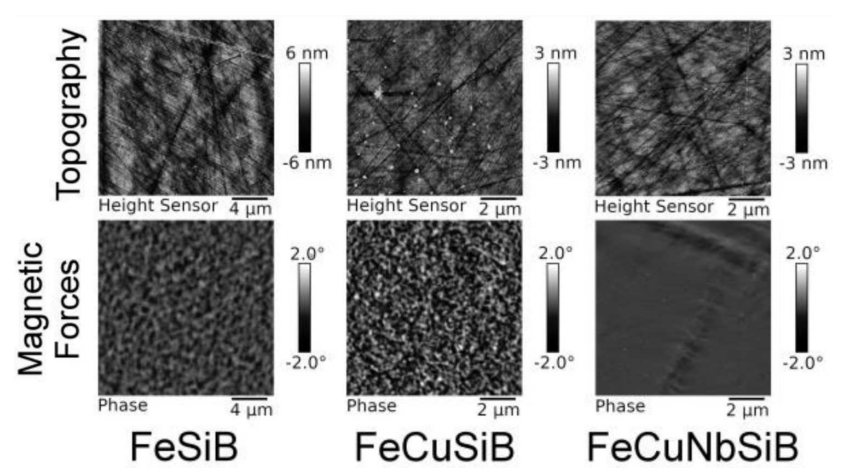

Fig. 11. Topography and magnetic forces images of Febased alloys after crystallization at $600^{\circ} \mathrm{C}$. 
Magnetic force measurements of the samples after conventional crystallization showed developed magnetic structure for all the surface of $\mathrm{FeSiB}$ and $\mathrm{FeCuSiB}$ alloys, as presented in Fig. 11. In turn, the alloy with niobium alloy addition, i.e., FeCuNbSiB, was magnetized only in selected areas. Apparently, annealing of samples at $600{ }^{\circ} \mathrm{C}$ deteriorates soft magnetic properties. Coercivity and remanence increased which enabled the observation of MFM signal. The alloy with niobium has unchanged coercivity and this is the reason of a very low magnetic signal, that could be detected only locally.

\section{Summary}

The aim of our wide studies is to find the best way to improve, from a technological point of view, magnetic properties of FeSi-based amorphous ribbons. Here, the changes of microstructure and magnetic properties caused by conventional heating and pulsed laser interference heating were presented and compared. PLIH produced periodically placed laser affected dots, where $\alpha-\mathrm{Fe}(\mathrm{Si})$ crystals were observed. On the other hand, conventional annealing of $\mathrm{FeSiB}$ alloy resulted in the creation of a coarse dendritic structure, which was largely refined in ribbons with alloy additions $(\mathrm{Cu}$ and $\mathrm{Nb}$ ). In those cases, nanocrystals appeared in the amorphous matrix. Magnetic hysteresis loops measurements of initial amorphous alloys showed that alloy additions decreased saturation magnetization. Conventional annealing resulted in magnetization lowering, in a similar way as laser heating did for $\mathrm{FeSiB}$ and $\mathrm{FeCuNbSiB}$. However, laser irradiation of $\mathrm{FeCuSiB}$ alloy resulted in a higher value of magnetization, increasing with the number of consecutive laser pulses. Coercivity of the alloys in the amorphous state as well as after laser heating, was about 20 Oe, while conventional crystallization led to coercivity doubling, except in the alloy with niobium addition. Magnetic force microscopy detects the magnetization on the sample surface only in the laser heated microareas. Conventional annealing led to strong magnetization of the entire sample surface, again except for FeCuNbSiB alloy. Since the MFM signal is caused by magnetic remanence of the observed surface, amorphous materials did not give any magnetic signal.

\section{Acknowledgments}

The authors would like to acknowledge financial support from the National Science Centre (NCN) of Poland (contract number: OPUS 10, UMO-2015/19/ B/ST8/01070).

\section{References}

[1] K. Suzuki, G. Herzer, Soft magnetic nanostructures and applications, in: Adv. Magn. Nanostructures Springer, 2006, pp. 365.

[2] R. Hasegawa, J. Magn. Magn. Mater. , 215 (2000).

[3] A. Wang, C. Zhao, H. Men, A. He, C. Chang, X. Wang, R.W. Li, J. Alloys Compd. 630, 209 (2015).

[4] Y. Yoshizawa, S. Oguma, K. Yamauchi, J. Appl. Phys. 64, (1988).

[5] G. Herzer, Nanocrystalline soft magnetic alloys, in: Handb. Magn. Mater. Elsevier Science, 1997, pp. 415.

[6] I. Garcia, N. Iturriza, J. Jose del Val, H. Grande, J.A. Pomposo, J. Gonzalez, J. Magn. Magn. Mater. 322, (2010).

[7] T. Jagielinski,IEEE Trans. Magn. 19, 1925 (1983).

[8] Y. Wu, K. Peng, L. Tang, W. Zhang, Intermetallics 91, 65 (2017).

[9] X. Zhen Fan, X. Wei He, R.K. Nutor, Ri-min Pan, J. Ju Zheng, H. Qun Ye, Feng-min Wu, J.Z. Jiang, Y. Zhang Fang, J. Magn. Magn. Mater. 469, 349 (2019).

[10] O. Czyż, J. Kusiński, A. Radziszewska, R. Ostrowski, J. Morgiel, J. Kanak, M. Kąc, Arch. Metall. Mater. 63, (2018).

[11] J. Kusinski, O. Czyz, A. Radziszewska, J. Morgiel, C. Kapusta, R. Ostrowski, M. Strzelec, K. Czyz, Int. J. Mater. Res. 110, (2019).

[12] M. Zheng, M. Yu, Y. Liu, R. Skomski, S.H. Liou, D.J. Sellmyer, V.N. Petryakov, Y.K. Verevkin, N.I. Polushkin, N.N. Salashchenko, Appl. Phys. Lett. 79, (2001).

[13] A. Lasagni, C. Holzapfel, F. Mücklich, Adv. Eng. Mater. 7, (2005).

[14] J. Marczak, A. Rycyk, A. Sarzyński, M. Strzelec, J. Kusiński, R. Major, Proc. Spie. 8703, (2013).

[15] W. Jia, Z. Peng, Z. Wang, X. Ni, C. Yue Wang, Appl. Surf. Sci. 253, (2006). 